Cárdenas Gutiérrez, A.R. y Montoro Fernández, E. (2017). Evaluación de un proyecto de educación emprendedora en la ESO. La visión del alumnado. Revista de Investigación Educativa, 35(2), 563-581

DOI: http://dx.doi.org/10.6018/rie.35.2.273221

\title{
Evaluación de un proyecto de educación emprendedora en la ESO. La visión del alumnado
}

\section{Assessment about a proyect in entrepreneurship education in the ESO.The student's view}

\author{
Dr. Antonio Ramón Cárdenas Gutiérrez y Elisabet Montoro Fernández \\ *Departamento de Teoría e Historía de la Educacion y Pedagogía Social. Facultad de Ciencias de la Educación. \\ Universidad de Sevilla (España)
}

\begin{abstract}
Resumen
Es una investigación mixta y descriptiva, teniendo como objetivos conocer el efecto en el potencial emprendedor del alumnado que ha participado en los programas ÍCARO y EJE, y comprender cómo las experiencias vividas han contribuido al desarrollo de los indicadores personales del potencial emprendedor. Cuantitativamente, participaron 182 estudiantes entre grupo control y experimental, contestando al test ATE. Cualitativamente, se seleccionaron a los 10 que más aumentaron su potencial emprendedor y a los 10 con menor puntuación. El efecto de los programas se evaluó mediante un análisis de la varianza con un diseño factorial $2 x 2$ con medidas repetidas y los datos se analizaron mediante una ANOVA mixto. La información de las entrevistas fue analizada mediante un análisis semiótico y narrativo. Según los datos cuantitativos no existe un claro efecto sobre el potencial emprendedor y las entrevistas desvelan que ambos programas parecen no configurarse adecuadamente para incidir en los indicadores personales del potencial emprendedor.

Palabras clave: educación emprendedora; evaluación; potencial emprendedor; educación secundaria obligatoria.

Correspondencia: Antonio Ramón Cárdenas Gutiérrez, acardenas1@us.es, Facultad de Ciencias de la Educación - C/ Pirotecnia s/n - 41013 Sevilla.

* Financiación del proyecto EDU2013-42936-P del Ministerio de Economía y Competitividad, dentro del Plan Estatal de Excelencia de Proyectos I+D.
\end{abstract}




\begin{abstract}
It is a mixed and descriptive research, having as aims knowing the effect on the enterprise potential of students who have participated in the programs ICARO and EJE, and understanding how having these experiences has helped the personal indicators development of the enterprise potential. Quantitatively, 182 students participated between control and experimental group, answering the ATE test. Qualitatively, the ten students who increased their enterprise potential more and the ten who got the lowest marks selected. The effect of the programs was evaluated via the analysis of the variance with a factorial design $2 \times 2$ with repeated measures and the data were analyzed through a mixed ANOVA. The information of the interviews was analyzed via a semiotic and narrative analysis. According to quantitative data, a relevant effect on enterprise potential does not exist and the interviews reveal that both programs seem not to be configured suitably to have an impact on the personal indicators of the enterprise potential.

Keywords: entrepreneurship education; assessment; enterprise potential; compulsory secundary education.
\end{abstract}

\title{
Introducción
}

La cumbre europea de Lisboa en el año 2000, supuso el punto de inicio del fomento de la cultura emprendedora en Europa. Así, con mayor o menor suerte, y mediante diversas estrategias (Education, Audiovisual and Culture Executive Agency, 2012) la Unión Europea ha ido favoreciendo la introducción de la educación emprendedora en los distintos sistemas educativos (EACEA, 2016). En el caso español, la legislación educativa paulatinamente ha incorporado la cultura emprendedora en la escuela (LOCE, 2002; LOE, 2006 y LOMCE, 2013), aunque de forma desigual según las etapas educativas y las comunidades autónomas (Global Entrepreneurship Monitor, 2014).

Marcada por sus orígenes economicistas (Katz, 2003), la educación emprendedora ha sido evaluada en función de los resultados o impacto que ha tenido en indicadores socioeconómicos (Millán, Congregado, Román, van Praag y van Stel, 2014: Walter y Block, 2016). Sin menospreciar su conveniencia, desde una perspectiva educativa, sería una evaluación irresoluta, ya que el potencial emprendedor, entendido como la suma de capacidades que una persona puede desarrollar para configurar su identidad emprendedora, quedaría un tanto difuminado. Las capacidades emprendedoras imbricadas en la dimensión personal del sujeto se encuentran íntimamente relacionadas con los procesos productivos. Aunque los factores externos facilitan la consolidación de la cultura emprendedora, no se entendería un aumento de la capacidad productiva y un desarrollo económico, social y laboral, sin contar con los factores personales, con el potencial emprendedor, cómo génesis de la actividad emprendedora (Edwards y Muir, 2012). Así, los estudios relacionados con las actitudes, los valores y la pasión por emprender son centrales en el desarrollo del potencial emprendedor (Murnieks, Mosakowski y Cardon, 2014).

La práctica de la educación emprendedora se relaciona con el desarrollo del emprendimiento económico y la formación en competencias (Martínez y Echeverría 2009). En este sentido, se han implementado un conjunto de Programas para fomentar la cultura emprendedora de los que sobresalen en Educación Secundaria EJE (Empresa 
Joven Europea) teniendo su origen en Asturias, dentro de Valnalón, e ÍCARO, en Andalucía. Nuestro interés se centra en estos programas por los siguientes motivos: son dos de los programas de mayor difusión a nivel nacional, ya que se aplican, de un modo $u$ otro, en seis comunidades autónomas. Ambos se desarrollan desde hace más de seis años siendo de los más longevos en su aplicación dentro de las escuelas y, sin embargo, apenas han sido objeto de evaluación educativa. Ambos programas poseen como finalidad que el alumnado cree y gestione su propia empresa mediante la fórmula jurídica de sociedades cooperativas, a través de las fases de creación, fabricación y venta de productos o servicios. Los dos programas configuran un Proyecto de Educación Emprendedora (en adelante PEE) para la ESO, intentando desarrollar en los adolescentes la creatividad, la autonomía, la motivación, el espíritu cooperativo y el liderazgo. Siendo éste nuestro objeto de estudio, pensamos que una educación emprendedora que asuma tales valores, supondría una inestimable aportación al crecimiento económico, pero también equivaldría como resorte para contrarrestar los riesgos de una educación emprendedora exclusivamente economicista.

\section{Planteamiento del problema y objetivos}

La Unión Europea plantea la necesidad de formar a los jóvenes en la competencia del espíritu emprendedor, aconsejando incorporar el emprendimiento en el contexto escolar en edades más tempranas. Sin embargo, esta directriz ha tenido escasa incidencia en las etapas de la educación obligatoria (Paço, Ferreira, Raposo, Rodrigues, y Dinis, 2011) en comparación con el ámbito universitario (Martin, McNally y Michael, 2013; Zhang, Duysters, Cloodt 2014). La reciente introducción del emprendimiento en el currículum dentro del ámbito escolar hace que la relación entre escuela y emprendimiento sea un campo inédito de estudio. Las incipientes investigaciones se centran prioritariamente en la evaluación e impacto de los programas de emprendimiento (Lepoutre, Van den Berghe, Tilleuil y Crijns, 2011; Oosterbeek, van Praag y Ijsselstein 2010). Si bien, el papel del docente en la implementación de la educación emprendedora empieza a ser tenido en cuenta (Ruskovaara y Pihkala, 2013), son exiguas las investigaciones que contemplan la perspectiva del alumnado en relación con el emprendimiento (Bernal y Cárdenas, 2014), aún teniendo en cuenta que son el objetivo principal sobre el que gira la educación emprendedora. Así pues, convendría indagar cómo se están llevando a cabo la implementación de la educación emprendedora, centrando nuestro interés en la dimensión personal de la educación emprendedora y, dejando a un lado, la tan consabida visión del emprendimiento entendido como mero desarrollo económico y empresarial. En este sentido, nos centramos en el potencial emprendedor comprendido educativamente como el conjunto de capacidades que podría desarrollar una persona y que facilitaría la construcción del dominio emprendedor de la identidad personal, esto es, su actitud o predisposición a iniciar proyectos personales. La voz del alumnado (Pomar y Pinya, 2015), nos permitirá estudiar con un carácter descriptivo y exploratorio este PEE de la ESO formado por los programas ÍCARO y EJE, con la intención de comprender cómo las experiencias educativas que ha vivido el alumnado, ha contribuido al desarrollo de los indicadores personales del potencial emprendedor de los sujetos estudiados. A este respecto, los objetivos que definimos son: 
a) Describir el efecto de un proyecto de educación emprendedora, formado por los programas ÍCARO y EJE, sobre el potencial emprendedor del alumnado y, para ello nos planteamos la siguiente hipótesis: la implementación de los programas ÍCARO y EJE incrementará el nivel del potencial emprendedor del alumnado partícipe.

b) Comprender, desde la óptica del alumnado, cómo se desarrollan los indicadores personales en función del potencial emprendedor, configurándolos en sus principales elementos desde una visión del emprendimiento personal.

\section{Método}

\section{Diseño}

Es una investigación mixta (Bericat, 1998; Mayoh y Onwuegbuzie, 2013) y descriptiva (Ato, López y Benavente, 2013), compuesta por una metodología cuantitativa y cualitativa. Se realizó un diseño bifase utilizándose la estrategia de combinación para integrar ambos métodos y se estableció que el método primario sería el cualitativo (QL) y el secundario el cuantitativo (qt) (Morgan, 1998), siendo la secuencia temporal cuantitativa y cualitativa (Johnson y Onwuegbuzie, 2004). Esto da como resultado el siguiente diseño (Creswell y Plano Clark, 2011): qt $\rightarrow$ QL.

Concretamente, se realizaron dos estudios, por un lado, un estudio de análisis de la varianza mediante un diseño factorial $2 \times 2$ con medidas repetidas antes y después (fase I) a un grupo control y experimental (León y Montero, 2003) y, por otro lado, un estudio de casos múltiples y extremos (Yin, 2009), mediante las entrevistas realizadas al alumnado seleccionado del grupo experimental (fase II) (Bisquerra, 2014; Woodsie, 2010) con mayor y menor puntuación en el test ATE (tabla 1). La validez de la investigación se realizó mediante una triangulación intermétodo combinando métodos cuantitativos y cualitativos (Denzin, 1970).

Tabla 1

Diseño multifase

\begin{tabular}{cccc}
\hline FASES & \multicolumn{2}{c}{ FASE I } & FASE II \\
\hline TÉCNICA & $\begin{array}{l}\text { PRETEST } \\
\text { (test ATE) }\end{array}$ & $\begin{array}{l}\text { POSTEST } \\
\text { (test ATE) }\end{array}$ & ENTREVISTAS \\
\hline MÉTODO & CUANTITATIVO & CUANTITATIVO & CUALITATIVO \\
\hline $\begin{array}{c}\text { PRIORIDAD Y } \\
\text { SECUENCIA }\end{array}$ & & & \\
\hline
\end{tabular}

Fuente elaboración propia 


\section{Población y Muestra}

Los sujetos fueron seleccionados mediante la técnica de muestreo intencional y opinático (Ruiz, 2012), según los criterios estratégicos personales de accesibilidad al campo de estudio, conocimiento previo de los informantes y puntuación obtenida a través del test ATE (Rodríguez, 2001). Para la realización del análisis cuantitativo, contamos con la participación de 182 estudiantes de la ESO pertenecientes a dos centros educativos sostenidos con fondos públicos, ambos de una línea educativa situados en Sevilla capital, uno como grupo control y otro como experimental, donde se implementó el PEE y posteriormente se realizó el estudio cualitativo (tabla 2).

Tabla 2

Muestra grupo control y experimental

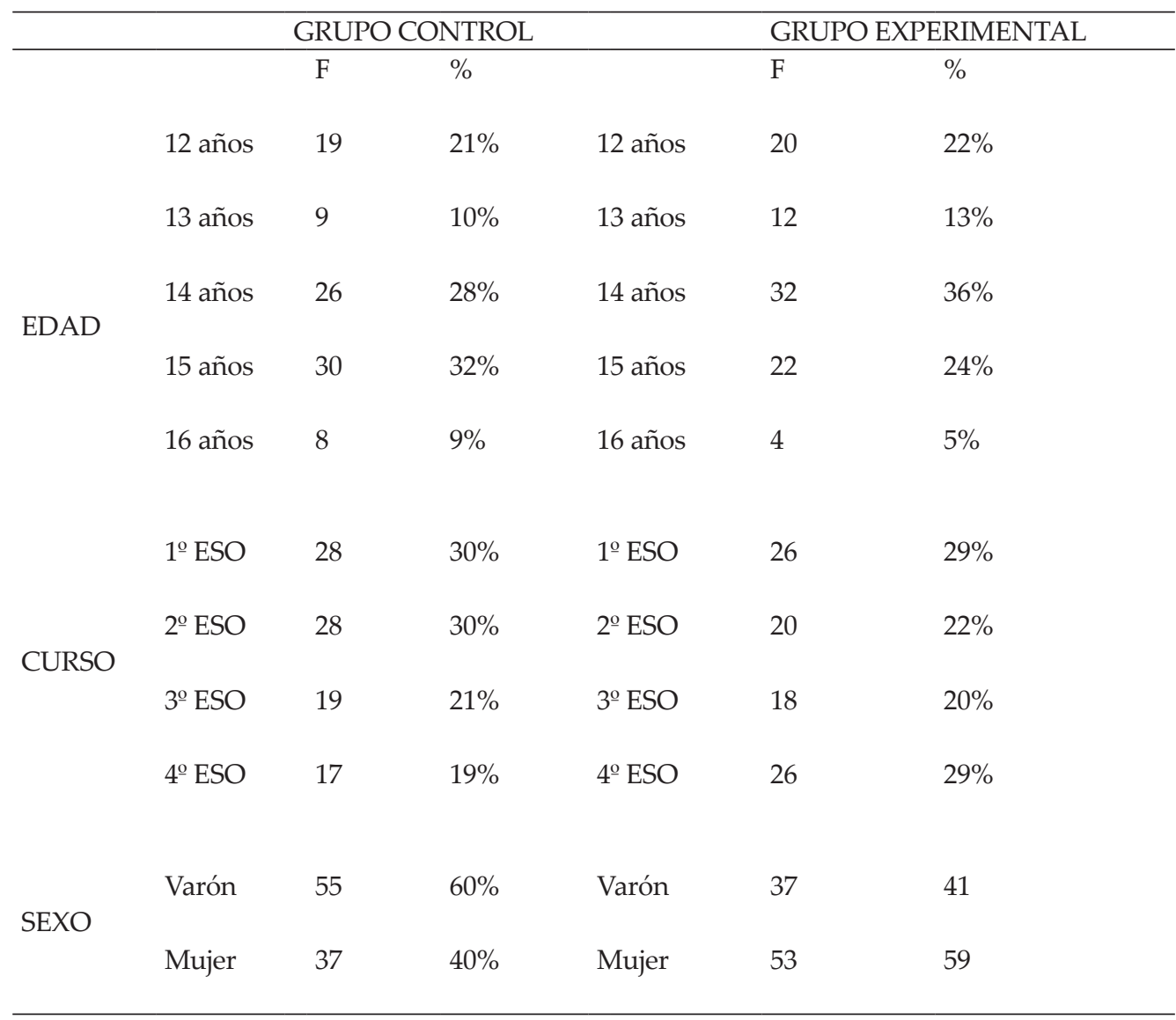

Fuente elaboración propia 
En relación al estudio cualitativo, de los 90 participantes en el grupo experimental se seleccionaron en función de las puntuaciones obtenidas en el test ATE a los 10 que más aumentaron su potencial emprendedor y a los 10 con menor puntuación, con la intención de contrastar mejor si existen posibles diferencias en los indicadores personales de emprendimiento en función de su mayor o menor potencial emprendedor.

\section{Instrumento}

Desde la perspectiva cuantitativa, el instrumento utilizado para la recogida de la información ha sido el Test ATE "Attitudes to Enterprise Test" (Athayde, 2009). Esta prueba mide el potencial emprendedor, esto es, la actitud o predisposición emprendedora de las personas. Su validez y fiabilidad ha sido reiteradamente contrastada (Steenekamp, Athayde y Van der Merwe 2011; Athayde, 2012). Este instrumento se compone de 5 subescalas que hacen referencia a 5 dimensiones del potencial emprendedor, además de la puntuación total en el constructo.

- Creatividad: capacidad de generar ideas que facilitan el proceso de innovación.

- Liderazgo: capacidad de construir equipos de trabajo, tomar decisiones, negociar y planificar.

- Logro: capacidad de perseverancia y de proactividad hacia la consecución de metas.

- Control Personal: capacidad para manejar y autorregular los comportamientos propios.

- Resolución de problemas: capacidad para afrontar circunstancias de incertidumbre e intensidad.

- Internamente, está formado por 6 ítems en cada escala, con una configuración total de 30 ítems con siete opciones de respuesta tipo Likert que van desde 1 (totalmente en desacuerdo) hasta 7 (totalmente de acuerdo).

Para la dimensión cualitativa (Tójar, 2006), utilizamos la entrevista en profundidad, semiestructurada (Corbin y Strauss, 2008; Kvale, 2011). La estructura interna está compuesta por los siguientes cinco bloques temáticos estudiados en el test ATE (tabla 3):

Utilizamos claves de identificación para clasificar las entrevistas (p.e.: S1PE+E12SV, quiere decir: S: sujeto; $\mathrm{PE}+/$ - mayor o menor potencial emprendedor; $\mathrm{E}$ : edad; $\mathrm{S}=$ sexo). La entrevista fue elaborada teniendo en cuenta aspectos esenciales de la evaluación de programas educativos y de los contenidos curriculares relacionados con la competencia básica en la autonomía e iniciativa personal (Casanova, 2012). La estructura final de la entrevista se elaboró a partir de una selección intencionada de cinco jueces expertos con experiencia en el diseño de programas educativos e investigación sobre emprendimiento (Banister, Burman, Parker, Taylor y Tindall, 2004). Para conseguir controlar los elementos espurios atendimos a los siguientes criterios metodológicos (tabla 4) (Guba, 1989; Gibbs, 2012). 
Tabla 3

Dimensiones de la entrevista

\begin{tabular}{ll}
\hline \multicolumn{1}{c}{ Dimensión } & \multicolumn{1}{c}{ Contenido } \\
Creatividad & $\begin{array}{l}\text { Cuestiones referidas al producto y al proceso creativo de la } \\
\text { persona. }\end{array}$ \\
Liderazgo & $\begin{array}{l}\text { Preguntas sobre la organización grupal y las habilidades sociales } \\
\text { de la persona. }\end{array}$ \\
Orientación al Logro & $\begin{array}{l}\text { Cuestiones para saber el grado de motivación y de actitud del } \\
\text { alumnado respecto a la práctica de los programas. }\end{array}$ \\
Control Personal & Preguntas para conocer el grado de autonomía del alumnado. \\
Resolución de problemas & $\begin{array}{l}\text { Cuestiones sobre cómo se ha enfrentado el alumnado a las } \\
\text { distintas dificultades en la aplicación de los programas. }\end{array}$ \\
\hline
\end{tabular}

Fuente elaboración propia.

Tabla 4

Criterios metodológicos

\begin{tabular}{lll}
\hline Credibilidad & $\bullet$ & Comprobaciones de los participantes. \\
Transferibilidad & Triangulación de intermétodo. \\
& - $\begin{array}{l}\text { Muestreo teórico (muestreo intencional). } \\
\text { Descripción minuciosa de los informantes recogida abundante } \\
\text { de los datos. }\end{array}$ \\
Dependencia & $\begin{array}{l}\text { Descripción de informantes por el número de sujeto, la edad } \\
\text { correspondiente y el sexo. }\end{array}$ \\
Confirmabilidad & Utilización del programa Atlas.ti. V7.0. \\
& $\begin{array}{l}\text { Comprobación con los sujetos entrevistados la información } \\
\text { recogida. }\end{array}$
\end{tabular}

Fuente elaboración propia.

\section{Procedimiento de recogida y análisis de datos}

La recogida de datos fue siempre voluntaria y garantizando el anonimato. En la fase cuantitativa, el alumnado realizó el test en sus aulas y en horario de tutorías, donde un miembro del grupo investigador explicó las instrucciones de cumplimentación con la presencia del tutor del curso correspondiente. El equipo investigador estaba formado 
por tres profesores de Universidad con experiencia en el diseño y evaluación de programas de educación emprendedora. Analizados los datos cuantitativos, seleccionamos a los informantes del grupo experimental, indicándoles la finalidad de las entrevistas. Éstas se realizaron en el Dpto. Orientación durante el horario escolar en función de una planificación previa de entrevistas que se consensuó con los profesores, para distorsionar lo menos posible la asistencia a la clase de los informantes. La recogida de datos se realizó en tres fases durante el curso 2015-2016 (tabla 5).

Tabla 5

Fases de recogida de datos

\begin{tabular}{|c|c|c|c|c|}
\hline FASES & MOMENTOS & RECOGIDA & MUESTRA & TÉCNICA \\
\hline 1 & Trimestre $1^{\circ}$ & $\begin{array}{c}\text { Pretest } \\
\text { Cuantitativo }\end{array}$ & $\begin{array}{l}\text { Grupo Control (92 sujetos) } \\
\text { Grupo Experimental (90 sujetos) }\end{array}$ & Test ATE \\
\hline 2 & Trimestre $2^{\underline{o}}$ & $\begin{array}{c}\text { Postest } \\
\text { Cuantitativo }\end{array}$ & $\begin{array}{l}\text { Grupo Control (92 sujetos) } \\
\text { Grupo Experimental (90 sujetos) }\end{array}$ & Test ATE \\
\hline 3 & Trimestre $3^{o}$ & $\begin{array}{l}\text { Entrevista } \\
\text { Cualitativa }\end{array}$ & $\begin{array}{l}10 \text { informantes con }+\mathrm{PE} \\
10 \text { informantes con }-\mathrm{PE}\end{array}$ & Entrevista \\
\hline
\end{tabular}

Fuente elaboración propia.

El análisis de datos se ha realizado mediante procedimientos cuantitativos y cualitativos. En la dimensión cuantitativa, para evaluar el efecto se empleó un análisis de la varianza mediante un diseño factorial $2 \times 2$ con medidas repetidas, donde el factor intergrupos es la participación o no en los programas ÍCARO y EJE; y siendo el factor intragrupo la medida repetida del tiempo transcurrido teniendo como niveles los dos momentos de medida (pretest y postest). El tamaño del efecto se obtuvo mediante el coeficiente eta-cuadrado parcial ( $\eta \mathrm{p} 2$ ) generalmente utilizado en investigación educativa (Sun, Pan y Wang, 2010). El tamaño del efecto se interpretó según el criterio de Cohen (1988): efecto pequeño $\eta p 2=.01-.06$, efecto medio $\eta p 2>.06-.14$ y efecto grande $\eta p 2.14$ $(\mathrm{d}=.80)$. Se comprobaron los supuestos de homocedasticidad con la prueba Levene y el supuesto de esfericidad con la prueba de Mauchly (Balluerka y Vergara, 2002). Los datos recabados se analizaron mediante un ANOVA mixto a través del programa estadístico SPSS versión 20. Las variables dependientes son las subescalas y puntuación total del ATE y las independientes los programas y la participación o no en ellos.

En las técnicas cualitativas, bajo un enfoque categórico del contenido (Ruiz, 2012), se realizó un análisis semiótico y narrativo (León y Montero, 2003), mediante la codificación abierta e inductiva de las narraciones del alumnado, consiguiendo un árbol de categorías emergente que paulatinamente se fue rediseñando (tabla 6) (Flick, 2012). El análisis del discurso se realizó contrastando las narraciones de los estudiantes seleccionados que más aumentaron su potencial emprendedor con los de menor puntuación (+/-PE). 
Tabla 6

Árbol de Categorías

\begin{tabular}{ll}
\hline & ÁRBOL DE CATEGORÍAS \\
\hline CATEGORÍAS & SUBCATEGORÍAS \\
\hline \multirow{2}{*}{ Proceso creativo } & Creación de ideas \\
& Planificación \\
& Sentido Crítico \\
& Visión de futuro \\
& Roles \\
& Conocimiento del grupo \\
Liderazgo & Habilidades sociales y comunicativas \\
& Tomas de decisiones \\
& \\
Orientación Motivadora & Motivación \\
& Desmotivación \\
& Satisfacción por los resultados \\
& Reconocimiento de éxito \\
& Satisfacción por el grado de libertad \\
Autonomía Personal & Responsabilidad e implicación \\
& Autoevaluación \\
Resolución de Problemas & Dificultades grupales \\
& Dificultades individuales \\
\end{tabular}

Fuente elaboración propia.

\section{Resultados}

\section{Resultados cuantitativos}

En los resultados del ANOVA mixto (tabla 7), se observa que no existe un efecto significativo del factor intergrupos, grupo control y experimental, sobre las variables dependientes, a excepción de la orientación al logro $\left(\mathrm{F}_{(1,120)}=6.858 ; p=.010\right)$ y control personal $\left(\mathrm{F}_{(1,120)}=4.225 ; p=0.41\right)$, ambas con un tamaño de efecto pequeño $(\eta p 2=.01$ .06). Esto nos indica la existencia de diferencias en las medias entre el grupo control y experimental en la evaluación inicial y final del Proyecto de Educación Emprendedora. Por otro lado, los datos desvelan que existe un efecto significativo del factor intragrupo sobre la variable dependiente de orientación al logro $\left(\mathrm{F}_{(1,120)}=3,849 ; p=0.5\right)$,

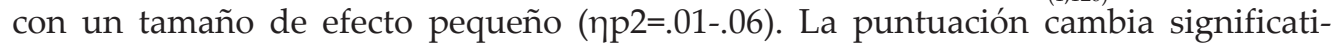
vamente con el tiempo en esta variable, según la participación del alumnado. Para 
saber si esta transformación se produce de manera similar en el grupo experimental y control o de forma diferente en alguno de ellos, observamos el efecto de interacción. Los contrastes grupo por momento de medida nos indican que no se produce efecto de interacción en ninguna de las variables estudiadas. En este caso, todo indicaría que los programas ÍCARO y EJE no han tenido efecto sobre el potencial emprendedor. Así pues, parece que estamos lejos de conseguir niveles adecuados en el desarrollo de la actitud emprendedora del alumnado.

Tabla 7

Resultados del ANOVA

\begin{tabular}{|c|c|c|c|c|c|c|c|c|c|}
\hline \multirow[b]{2}{*}{ VARIABLES } & \multicolumn{3}{|c|}{ Efecto Intergrupos } & \multicolumn{3}{|c|}{ Efecto Intragupos } & \multicolumn{3}{|c|}{ Efecto Interacción } \\
\hline & F & $\mathrm{P}$ & $\eta p 2$ & $\mathrm{~F}$ & $\mathrm{P}$ & $\eta p 2$ & F & $\mathrm{P}$ & $\eta p 2$ \\
\hline Creatividad & 0,222 & 0,638 & 0,001 & 0,084 & 0,773 & 0,000 & 1,358 & 0,210 & 0,007 \\
\hline Liderazgo & 0,577 & 0,448 & 0,003 & 0,216 & 0,642 & 0,001 & 0,046 & 0,831 & 0,000 \\
\hline $\begin{array}{l}\text { Resolución de } \\
\text { Problemas }\end{array}$ & 0,522 & 0,471 & 0,003 & 0,640 & 0,048 & 0,003 & 0,200 & 0,655 & 0,001 \\
\hline $\begin{array}{l}\text { Orientación al } \\
\text { Logro }\end{array}$ & 6,858 & $0,010^{*}$ & $0,034^{*}$ & 3,849 & $0,050^{*}$ & 0,020 & 2,601 & 0,108 & 0,013 \\
\hline Control Personal & 4,225 & $0,041^{*}$ & $0,022^{*}$ & 0,368 & 0,545 & 0,002 & 2,568 & 0,111 & 0,013 \\
\hline Total & 1,935 & 0,166 & 0,010 & 0,719 & 0,398 & 0,004 & 1,511 & 0,220 & 0,008 \\
\hline
\end{tabular}

*Significación estadística

Fuente elaboración propia

\section{Resultados cualitativos}

\section{Proceso creativo}

En la creación de ideas, el alumnado con +/-PE, comentan que "la profesora nos daba ideas y nosotros escogíamos" (S8PE+E12SM). Así, la profesora era quien sugería diversas ideas y sobre ellas tenían que debatir y seleccionar las más adecuadas. De este modo, el alumnado realizó un proceso mimético sin generar ideas nuevas, dejando la innovación a un lado (Schimdt, Soper y Bernaciak, 2013).

En relación con los procesos de planificación necesarios para desarrollar las ideas, encontramos diferencias entre el alumnado con +/-PE. El alumnado con +PE comenta: "nos dividimos el trabajo en fases y nos marcamos plazos para trabajar bien los productos" (S32PE+E13SV), esto es, se dedicaron a dividir la elaboración del producto en fases, marcándose plazos de tiempo que debían de cumplir con rigurosidad. Por el 
contrario, los de-PE creyeron innecesario fijar unos plazos de tiempo. Estas diferencias de planificación llevaron a mejores y peores resultados de fabricación.

Ambos tipos de alumnado vivieron de forma diferente el proceso creativo (Fillis y Rentscheler, 2010; López y Navarro 2010). Así pues, en la subcategoría de sentido crítico, los que aumentaron aseguraron tener dudas durante todo el proceso, cuestionándose las ideas propias y ajenas, además de reconocer que los productos podrían mejorarse. En cambio, los que disminuyeron afirman: "no tuvimos dudas, íbamos haciendo las cosas y ya está (...) no comparamos nuestros productos" (S42PE-E12SM).

En la subcategoría de visión de futuro, el alumnado con +/-PE coincidían en volver a participar en este PEE en próximos cursos: "me gustaría tener la oportunidad de crear cosas y tener esta libertad en otras asignaturas, (...) aunque se tiene que mejorar la organización" (S45PE-E13SM).

\section{Liderazgo}

Respecto a los roles, en general, el alumnado expuso que no establecieron explícitamente ningún tipo de rol y que no había un líder, "hacíamos el trabajo entre todos y tomábamos las decisiones en conjunto, poniéndonos de acuerdo" (S24PE-E12SM). Sin embargo, reconocen algunos casos en los que había compañeros que sí dirigían más el trabajo, sin llegar a considerarlos líderes del grupo. De esta manera, carecían de una estructura organizativa que delimitara las responsabilidades de cada uno de los miembros de las cooperativas (Puchol, 2012).

En cuanto al conocimiento del grupo, parece ser que todos se conocían bastante bien e incluso sabían qué cosas se les daba mejor o peor a sus compañeros. Como dice el estudiante: "sí, nos conocíamos bien y sabíamos quién podría hacer tal cosa y quién no" (S41PE-E16SV). No obstante, existen diferencias. El alumnado con +PE pensaba que "es importante conocernos para trabajar juntos" (S10PE+E13SM), mientras que los de -PE creían que no era tan significativo, puesto que argumentaban que el trabajo poco tenía que ver con las relaciones personales: "nos conocíamos bien, pero yo creo que tampoco eso es tan importante, porque para trabajar no tenemos que ser los mejores amigos" (S18PE-E14SV). Las habilidades sociales y comunicativas son facilitadoras del trabajo cooperativo y claves para ejercer un buen liderazgo (Van Hemmen, Urbano y Álvarez, 2013). No obstante, una gran mayoría del alumnado independientemente del +/-PE confirma que no ha sabido negociar sus ideas, ni expresar sus pensamientos, llegando a veces a imponer sus criterios de forma autoritaria, como recuerda un estudiante: "algunas veces, yo no me podía explicar bien o ellos no me entendían. Y me enfadaba tanto que decía esto es así y punto" (S37PE-E12SM).

Aunque, la mayoría del alumnado narró haber intentado promover la participación: "les decía que venga, que hicieran cosas, que podíamos hacerlo" (S30PE+E13SV). Sin embargo, todos coinciden en su incapacidad para generarla entre sus compañeros: "no era capaz de hacer que participaran"(S30PE+E13SV). Parece ser que al alumnado le faltaría ejercer correctamente las competencias interpersonales tan necesarias para un adecuado liderazgo (Palomo, 2013).

La toma de decisiones se hacía de forma conjunta por parte del alumnado, pero los estudiantes de +/-PE reconocieron que les "costaba mucho trabajo tomar las decisio- 
nes" (S13PE+E12SV), más aún cuando las consideraban difíciles, y que "preferían que las decisiones las tomen los demás" (S23PE-E15SV), sintiéndose más cómodos y tranquilos cuando delegaban las decisiones. Según Hammond, Keeney y Raiffa (2007), todo indicaría que el alumnado no está suficientemente familiarizado con la toma de decisiones de forma responsable dentro de la práctica educativa.

\section{Orientación motivadora}

Partiendo de la voluntariedad de participación en el PEE, ambos tipos de alumnado convergen en una alta motivación por empezar las cooperativas, incluso con más ilusión que ediciones anteriores: "me ha gustado más, porque el año pasado no teníamos tanta libertad” (S39PE+E12SM). Así pues, desde un primer momento, parece que mantenían una actitud positiva a la hora de enfrentarse al trabajo en las cooperativas. Esa motivación inicial es relevante, pues la desmotivación del alumnado condiciona, en buen grado, el aprendizaje y la práctica educativa (Nuñez, Fontana y Pascual, 2011).

Sin embargo, el alumnado con $+\mathrm{PE}$ afirma que se sentían motivados "porque teníamos que construir cosas nuevas, y aunque parecían difíciles también eso nos motivaba" (S1PE+E16SV). Por el contrario, el alumnado con -PE alega que estaban motivados "cuando los productos eran fáciles de hacer, hasta que se complicaban las cosas y tareas" (S9PE-E13SV). Así pues, en ambos tipos de estudiantes se distingue la relación entre el nivel de motivación y el grado de dificultad de las tareas que han realizado (Schunk, y Zimmerman, 2008).

Además, cabe destacar factores externos e internos (temperatura, hora de clase, clima del grupo o estados de ánimo) como desencadenantes de la desmotivación hacia las cooperativas: "si algún día tenías un examen o un problema, o venías de la clase de Educación Física teníamos menos ganas"(S11PE+E15SM). En esos casos, convendría realizar estrategias educativas que impulsen el despertar de la motivación perdida (Marina, 2013).

Por otro lado, existen diferencias en el grado de satisfacción en relación con los resultados alcanzados en las cooperativas, pues el alumnado con $+\mathrm{PE}$ se mostró satisfecho con sus productos, con el grupo de trabajo y con sus tareas, cumpliéndose de esta forma las expectativas que tenían en un principio, aunque es necesario mencionar que una minoría de este alumnado afirmó no sentirse plenamente satisfecho porque pensaban que podrían haberlo hecho bastante mejor: "me sentí satisfecho tanto con los productos como con la forma de trabajar del grupo" (S8PE+E12SM). En cambio, los de -PE, aunque, en un primer momento, sintieron cierto grado de satisfacción, aseguraron que los resultados y el funcionamiento del grupo eran mejorables, como afirma un estudiante: "las cooperativas se podrían haber hecho bastante mejor (...) los productos, las tareas, la venta, todo en general (...) mi grupo no funcionaba bien"(S41PE-E16SV).

Las narraciones relacionadas con el reconocimiento del trabajo realizado, tales como: "la profesora nos felicitó por nuestro trabajo y los compañeros nos dijeron que nuestros productos eran muy chulos" (S30PE-E14SV) o "nos sentimos orgullosos, porque había merecido la pena tanto esfuerzo" (S32PE+E13SV), desvelan que ambos tipos de estudiantes tenían la necesidad de que el resto de personas les reconocieran su buena labor, hecho que les hacía sentir muy bien consigo mismos. 


\section{Autonomía Personal}

EJE e ÍCARO se caracterizan por ser prácticas educativas flexibles y con espacios de libertad para el alumnado, favoreciendo un mejor desarrollo de la autonomía y control personal (Helwing, 2006; Aviram y Yonah, 2004). A este respecto, el alumnado con +PE estaba satisfecho con el grado de libertad que tenía para desarrollar el trabajo: "me sentía bien porque tenía libertad para hacer lo que quisiera. Hacer lo que uno cree que es necesario estuvo muy bien" (S1PE+E16SV). Por el contrario, el alumnado con -PE reconoció haberse sentido nervioso e inquieto al no tener instrucciones específicas de cómo realizar el trabajo. Así lo narra una alumna: "me chocaba un poco no tener instrucciones, me ponía nerviosa, nunca en el colegio me habían dejado hacer tanto sin indicaciones, la verdad no estoy acostumbrada" (S42PE-E12SM). Según las narraciones parece que existen grados de autonomía en función del PE del alumnado.

Sin duda alguna, a mayor grado de libertad, se amplían los márgenes para ejercer la autonomía y la responsabilidad (Veugelers, 2011). Respecto a la responsabilidad e implicación en las tareas, encontramos que el alumnado con $+\mathrm{PE}$ fue más responsable y participó en mayor medida que aquellos estudiantes con -PE. La narración de un estudiante nos describe esta dicotomía: "fuimos responsables con las tareas y participamos en todas porque no nos parecía justo que muchos trabajasen mucho y otros poco $(. .$.$) había gente que no se responsabilizaba por sí mismo de nada, a veces era$ desolador" (S2PE+E14SV).

En este sentido, en cuanto al establecimiento de reglas o normas, la mayoría del alumnado con $+\mathrm{PE}$ afirmó haber fijado reglas desde el principio. Sin embargo, algunos de ellos narraron que no consiguieron "seguir las normas, sobre todo, pasado algún tiempo, aunque no sé las causas de esta dejadez " (S2PE+E14SV). Por el contrario, el alumnado con -PE, en general, señaló que no lo consideraron necesario: "no pusimos reglas cuando se empezó con las cooperativas, no caímos en eso, la verdad (...) todo se realizaba de manera caótica" (S9PE-E13SV).

Las mismas diferencias se dan cuando les pedimos que realicen una autoevaluación, ya que el alumnado con $+\mathrm{PE}$ comenta que se encontraban "alegres y optimistas con el trabajo. No nos desanimábamos, aportábamos soluciones. Intentábamos continuar con los productos" (S2PE+E14SV). No obstante, parece ser que incluso aquellos estudiantes con +PE no lograron solucionar muchos de los problemas que tuvieron, aunque lo intentaron no alcanzaron resultados. Así pues, según las narraciones, el alumnado con + PE se siente más autónomo, responsable y cómodo con los márgenes de libertad que les ha ofrecido este PEE. En otro sentido, el alumnado con -PE expresó que en bastantes ocasiones "se agobiaban mucho, que no les daba tiempo a solucionar todos los problemas (...) nos sentíamos muy presionados" (S18PE-E14SV). Todo apuntaría a que un menor potencial emprendedor estaría relacionado con carencias relacionadas con el manejo de situaciones de presión.

\section{Resolución de problemas}

Ambos tipos de estudiantes coinciden en haber tenido dificultades grupales de diversa tipología: baja calidad en los productos, escasez de materiales, mala organización del 
tiempo o discusiones y peleas entre los miembros del grupo. Sin embargo, el afrontamiento de los problemas diverge entre el alumnado. Parece ser que el alumnado con + PE consiguió tener una mayor capacidad de resolución de problemas, pues se detuvieron en analizar la utilidad del producto, valorando las ventajas e inconvenientes, y se anticiparon a determinados problemas que podrían aparecer en el transcurso del trabajo, como dice una alumna: "sí, analizamos las ventajas e inconvenientes para saber si el producto sería útil o no" (S11PE+E15SM). Por otro lado, el alumnado con -PE no consideró necesario analizar las ventajas o inconvenientes de un producto y tampoco fue capaz de anticipar problema alguno porque iban actuando sobre la marcha. Así lo recuerda un estudiante: "no, cuando salieron problemas no los habíamos pensado antes. Íbamos actuando sobre la marcha" (S30PE-E14SV).

Además, el alumnado en general reconoció haber tenido grandes dificultades individuales para aportar soluciones o alternativas que consiguieran paliar los conflictos. Tanto es así, que la mayoría afirma que no lo hubiesen conseguido sin contar con el asesoramiento de la profesora, las narraciones de dos alumnos así lo desvelan: "intentamos solucionarlo entre nosotros, a veces lo conseguíamos y otras tenía que venir la profesora para que nos ayudara" (S7PE-E13SM) o "normalmente teníamos que pedirle soluciones a la profesora, no éramos capaces de ponernos de acuerdo"(S11PE+E15SM). La eficacia del aprendizaje cooperativo dentro del aula es compleja, pues se aleja de la mera trasmisión de conocimientos, el alumnado y el profesorado adquieren nuevos roles difíciles de desempeñar correctamente sin unas bases construidas desde los primeros años de escolarización (Torrego y Negro, 2012).

\section{Discusión y conclusiones}

Los efectos del PEE sobre el potencial emprendedor del alumnado son inapreciables, ya que no se producen efectos de interacción en ninguna de las variables, sugiriendo que los programas ÍCARO y EJE no han incrementado el nivel de potencial emprendedor del alumnado participante, como cabría esperar tras la participación en ellos.

El contraste argumentativo del alumnado con +/-PE, nos ofrece un acercamiento comprensivo de lo que acontece en la práctica educativa. En este sentido, encontramos que el bajo desarrollo de los indicadores personales sería atribuible a ciertos elementos que, de una forma u otra, han ido mermando la práctica educativa emprendedora.

Así pues, este PEE no parece fomentar adecuadamente la creatividad, ya que la creación de productos se ha reducido a procesos rutinarios de producción sin la creación de ideas propias que generaran unos resultados de producción originales. Las narraciones nos desvelan las carencias organizativas y de recursos en el proceso creativo que lastrarían con creces el correcto desarrollo de la creatividad (Stemberg, 1999).

Todo apuntaría a que no han existido criterios correctos de agrupamiento por parte de los participantes o de los docentes responsables del Proyecto de Educación Emprendedora. En las entrevistas, se entrevé que ÍCARO y EJE se han desarrollado a través de meros agrupamientos, más que de un serio trabajo cooperativo, desde el cual se pudiera ejercer un liderazgo personal. A pesar de que las relaciones personales han sido correctas, la falta de capacidad negociadora, la incapacidad de abrir canales de negociación entre el alumnado cuando surgían problemas o la carencia de una toma de 
decisiones por falta de información y conocimiento, pone en entredicho el desarrollo del liderazgo personal en este PEE (Bolívar, 2010).

La participación voluntaria es una garantía de alta motivación intrínseca por parte del alumnado (Urcola, 2008). Si bien, ha existido una alta motivación inicial, no es menos cierto que esa motivación ha ido disminuyendo durante el desarrollo del Proyecto de Educación Emprendedora. Las causas, según las narraciones del alumnado, han sido: grado de dificultad de las tareas encomendadas, horario de implementación de los programas, el cansancio físico y mental al realizarse los programas en horas de tutoría o la incidencia de factores situacionales del aula. Todas ellas en su conjunto han provocado que las expectativas motivacionales iniciales se fueran reduciendo paulatinamente.

La autonomía y el emprendimiento entendidos como sinónimos, tienen en común el grado de control personal que posee el sujeto para realizar un determinado proyecto (Organización para la Cooperación y el Desarrollo Económicos, 2005). En este sentido, el alumnado considera como muy positivo el grado de libertad que tuvieron para implementar las tareas y actividades, en comparación con otras áreas curriculares. Sin embargo, parece ser que ese espacio de libertad no supieron ocuparlo adecuadamente, sobre todo, no fueron capaces de ejercer un grado adecuado de responsabilidad y control en la realización de las tareas y actividades. Estas carencias, tal vez, provienen de la falta de experiencia del ejercicio de la libertad dentro del ámbito educativo (Puig y Martín, 2007), tanto por parte del alumnado como del profesorado.

Según las entrevistas, en la resolución de problemas no se ha incidido adecuadamente en la implementación de ÍCARO y EJE. El alumnado nos cuenta que fueron incapaces de resolver un amplio número de problemas que surgieron en la implementación de las cooperativas. La mayoría de esos problemas se encuentran relacionados con la producción de los servicios o productos, la gestión de los plazos fijados en la planificación y los recursos utilizados. Sin embargo, latente a todos ellos se encuentra la incapacidad de resolver los conflictos entre iguales de manera autónoma y responsable. En bastantes ocasiones, el profesor tuvo que realizar una labor de mediador y facilitador de las relaciones sociales, ante la falta de consenso entre el propio alumnado para llegar a los objetivos marcados.

En general, se entrevé que ambos programas no se encuentran suficientemente desarrollados para influir adecuadamente sobre la identidad emprendedora del alumnado. Los resultados del test ATE nos permiten ver el nulo efecto o incidencia del programa sobre el ámbito emprendedor de la identidad, y la riqueza de matices de las narraciones del alumnado con +/- PE nos desvelan una práctica de educación emprendedora con escasa organización y planificación. Así pues, todo indicaría que este PEE se implementa de una forma un tanto azarosa, perfilándose más bien como una declaración de intenciones y propuestas educativas que se viene implementando con mayor o menor acierto, pero sin una incidencia clara sobre los indicadores personales del emprendimiento.

Si bien, los datos de esta investigación arrojan luz sobre lo que está sucediendo en la aplicación y efecto de estos programas de educación emprendedora, sería conveniente extender la investigación a una muestra de alumnado más amplia y contar con una diversidad de centros educativos que nos permita reflexionar sobre la implementación 
de este tipo de programas con una visión más completa. Aunque, no es menos cierto, que los resultados obtenidos nos aportan una serie de inéditas cuestiones sobre la práctica de la educación emprendedora: cómo se organiza los programas de educación emprendedora, qué tipo de metodología se está utilizando en la implementación de los programas, si existen materiales curriculares adecuados, qué formación posee el profesorado en esta materia, si se está realizando una evaluación de los aprendizajes, entre otras. En definitiva, un elenco de sugerentes interrogantes que abre el abanico de posibilidades de estudios evaluativos que amplíen el escaso conocimiento que se tiene sobre lo qué está ocurriendo con la educación emprendedora en la escuela (Diego y Vega, 2015).

\section{Referencias}

Athayde, R. (2009). Measuring enterprise potential in young people. Entrepreneurship Theory and Practice, 33(2), 481-500. doi: 10.1111/j.1540-6520.2009.00300.x

Athayde, R. (2012). The impact of enterprise education on attitudes to enterprise in young people: an evaluation study. Education +Training, 54(8/9), 709-726. doi: 10.1108/00400911211274846

Ato, M., López, J. \& Benavente, A. (2013). Un sistema de clasificación de los diseños de investigación en psicología. Anales de Psicología, 29(3), 1038-1059. doi: 10.6018/ analesps.29.3.178511

Aviram, R. \& Yonah, Y. (2004). Flexible control: Towards a conception of personal autonomy for postmodern education. Educational Philosophy and Theory, 36(1), 3-17. doi: 10.1111/j.1469-5812.2004.00045.x

Balluerka, N. \& Vergara, A. J. (2002). Diseños de investigación experimental en psicología. Madrid: Prentice Hall.

Banister, P., Burman, E., Parker, I., Taylor, M. \& Tindall, C. (2004). Métodos Cualitativos en Psicología: Una Guía Para la Investigación. Guadalajara: Universidad de Guadalajara.

Bericat, E. (1998). La integración de los métodos cuantitativo y cualitativo en la investigación social. Barcelona: Ariel.

Bernal, A. \& Cárdenas, A. (2014). La formación de emprendedores en la escuela y su repercusión en el ámbito personal. Una investigación narrativa centrada en el Programa EME. Revista Española de Pedagogía, 72(257), 124-143.

Bisquerra, R. (2014). Metodología de la investigación educativa. Madrid: La Muralla.

Bolívar, A. (2010). ¿Cómo un liderazgo pedagógico y distribuido mejora los logros académicos? Revisión de la investigación y propuesta. Magis, Revista Internacional de Investigación en Educación, 3(5), 79-106.

Casanova, A.M. (2012). La evaluación de las competencias básicas. Madrid: La Muralla.

Cohen, J. (1988). Statistical Power Analysis for the Behavioral Sciences. New Jersey: Lawrence Erlbaum Associates.

Corbin, J. \& Strauss, A. (2008). Basics of Qualitative Research: Techniques and Procedures for Developing Grounded Theory (3rd ed.). Thousand Oaks, CA: Sage.

Creswell, J.W. \& Plano Clark, V.L. (2011). Designing and Conducting Mixed Methods Research. London: Sage. 
Denzin, N.K. (1970). Sociological Methods: a Source Book. Chicago: Aldine Publishing Company. doi: 10.1016/j.jbusvent.2012.03.002

Diego, I. \& Vega, J. A. (2015). La educación para el emprendimiento en el sistema educativo español. Año 2015. Estudio rediE. Madrid: Ministerio de Educación, Cultura y Deporte.

EACEA. (2012). Entrepreneurship Education at School in Europe. National Strategies, Curricula and Learning Outcomes. Bruselas: Education, Audiovisual and Culture Executiva Agency. doi:10.2797/80384

EACEA. (2016). Entrepreneurship in Europe Eurydice Report Education at School. Luxembourg: Publications Office of the European Union. doi:10.2797/301610

Edwards. L.J. \& Muir, E.J. (2012). Evaluating enterprise education: why do it?. Education + Training, 54 (4), 278-290. doi:10.1108/00400911211236136

Fillis, I. \& Rentschler, R. (2010). The role of creativity in entrepreneurship. Journal of Enterprising Culture, 18(1), 49-81. doi: 10.1142/S0218495810000501

Flick, U. (2012). Introducción a la investigación cualitativa. Madrid: Morata.

Gibbs, G. (2012). El análisis de datos cualitativos en investigación cualitativa. Madrid: Morata.

Global Entrepreneurship Monitor (2014). Informe GEM España 2014. Santander: Universidad de Cantabria.

Guba, E.G. (1989). Criterios de credibilidad en la investigación naturalista. En J. Gimeno Sacristán \& A. Pérez Gómez (Eds.), La enseñanza: su teoría y su práctica (3ª ed., pp. 148-165). Madrid: Akal.

Hammond, J.S., Keeney, R.L. \& Raiffa, H. (2007). Decisiones inteligentes: guía práctica para tomar mejores decisiones. Barcelona: Gestión 2000.

Helwig, C.C. (2006). The development of personal autonomy throughout cultures. Cognitive Development, 21, 458-473. doi: 10.1016/j.cogdev.2006.06.009

Johnson, B. \& Onwuegbuzie, A. (2004). Mixed Methods Research: A research paradigm whose time has come. Educational Research, 33(7), 14-26.

Katz, J.M. (2003). The chronology and intellectual trajectory of American entrepreneurship education 1876-1999. Journal of Business Venturing, 18 (2), 283-300. doi:10.1016/ S0883-9026(02)00098-8

Kvale, S. (2011). Las entrevistas de investigación cualitativa. Madrid: Morata.

León, O.G. \& Montero, I. (2003). Métodos de investigación en Psicología y Educación. Madrid: McGraw-Hill.

Lepoutre J., Van den Berghe W., Tilleuil O. \& Crijns H. (2011). A new approach to testing the effects of entrepreneurship education among secondary school pupils. En: M. Raposo, D. Smallbone, K. Balaton \& L. Hortoványi (Eds.), Entrepreneurship, Growth and Economic Development. Frontiers in Europeaen Entrepreneuship Research (pp.94-117). Cheltenham: Edward Elgar Publishing Limited.

Ley Orgánica 5/2002, de 19 de junio, de las Cualificaciones y de la Formación Profesional. BOE 147 de junio.

Ley Orgánica 2/2006, de 3 de mayo, de Educación. BOE 106 de mayo.

Ley Orgánica 8/2013, de 9 de diciembre, para la Mejora de la Calidad Educativa. BOE 295 de diciembre.

López, O. \& Navarro, J. (2010). Creatividad e inteligencia: un estudio en Educación Primaria. Revista de Investigación Educativa, 28(2), 283-296. 
Marina, J.A. (2013). Talento, Motivación e Inteligencia. Las claves para una educación eficaz. Barcelona: Ariel.

Martin, B.C., McNally, J.J. \& Michael J.K. (2013). Examining the formation of human capital in entrepreneurship: A meta-analysis of entrepreneurship education outcomes. Journal of Business Venturing, 28(2), 211-224.

Martínez, P. \& Echeverría, B. (2009). Formación Basada en Competencias. Revista de Investigación Educativa, 27(1), 125-147.

Mayoh, J. \& Onwuegbuzie, A. (2013). Toward a Conceptualization of Mixed Methods Phenomenological Research. Journal of Mixed Methods Research, 9(1), 91-107. doi: $10.1177 / 1558689813505358$

Millán, J.M., Congregado, E., Román., C., van Praag, M. \& van Stel, A. (2014).The value of an educated population for an individual's entrepreneurship success. Journal of Business Venturing, 29 (5), 612-632. doi: 10.1016/j.jbusvent.2013.09.003

Morgan, D.L. (1998). Practical strategies for combining qualitative and quantitative methods: Applications to health research. Qualitative Health Researchs, 8(3), 326-376. doi: 10.1177/104973239800800307

Murnieks, Ch.Y., Mosakowski, E. \& Cardon, M. S. (2014). Pathways of Passion: Identity Centrality, Passion, and Behavior Among Entrepreneurs. Journal of Management, 40(6), 1583-1606. doi: 10.1177/0149206311433855

Nuñez, M.C., Fontana, M. \& Pascual, I. (2011). Estudio exploratorio de las características motivacionales del alumnado de la ESO y su relación con las expectativas de rendimiento académico. Electronic Journal of Research in Educational Psychology, 9(1), 357-382.

OCDE, (2005). The Definition and Selection of Key Competences. Executive Summary. París: OCDE.

Oosterbeek, H., van. Praag, M. \& Ijsselstein, A. (2010). The impact of entrepreneurship education on entrepreneurship skills and motivation. European Economic Review, 54(3), 442-454. doi:10.1016/j.euroecorev.2009.08.002

Paço, A.M.F., Ferreira, J. M., Raposo, M., Rodrigues, R. G. \& Dinis, A. (2011). Behaviours and entrepreneurial intention: empirical findings about secondary students. Journal of International Entrepreneurship, 9(1), 20-38. doi 10.1007/s10843-010-0071-9

Palomo, M.T. (2013). Liderazgo y motivación de equipos de trabajo. Madrid: ESIC.

Pomar, M.I. \& Pinya, C. (2015). The voice of youths about school and its mark on their lives. Improving Schools, 18(2), 111-126. doi: 10.1177/1365480215589662

Puchol, L. (2012). Dirección y Gestión de RRHH. Madrid: Díaz de Santos.

Puig, J.M. \& Martín, X. (2007). Competencia en autonomía e iniciativa personal. Madrid: Alianza Editorial.

Rodríguez, J. (2001). Método de muestreo. Madrid: CIS.

Ruiz, J.I. (2012). Metodología de la Investigación Cualitativa. 5ª edición. Universidad de Deusto: Bilbao

Ruskovaara, E. \& Pihkala, T. (2013). Teachers implementing entrepreneurship education: classroom practices. Education+Training, 55(2), 204-216. doi: 10.1108/00400911311304832

Schmidt, J.J., Soper, J.C. \& Bernaciak, J. (2013). Creativity in the entrepreneurship program: a survery of the directors of award winning programas. Journal of Entrepreneurship Education, 16, 31-44. 
Schunk, D.L. \& Zimmerman, B.J. (2008). Motivation and Self-Regulated Learning: Theory, Research, and Applications. New York: Lawrence Erlbaum Associates.

Steenekamp, A. G., Athayde, R. \& Van der Merwe, S.P. (2011). An investigation into youth entrepreneurship in selected South African secondary schools: an exploratory study. Southern African Business Review, 15(3), 46-75.

Stemberg, R. (1999). Hanbook of creativity. New York. Cambridge University Press.

Sun, S., Pan, W. \& Wang, L. L. (2010). A comprehensive review of effect size reporting and interpreting practices in academic journals in education and psychology. Journal of Educational Psychology, 102(4), 989-1004. doi: 10.1037/a0019507

Tójar, J.C. (2006). Investigación cualitativa. Comprender y actuar. Madrid: La Muralla.

Torrego, J. C. \& Negro, A. (2012). Aprendizaje cooperativo en las aulas: Fundamentos y recursos para su implantación. Madrid: Alianza Editorial.

Urcola, J.L. (2008). La motivación empieza en uno mismo. Madrid: ESIC Editorial.

Van Hemmen, S., Urbano, D. \& Álvarez, C. (2013). Charismatic leadership and entrepreneurial activity: An empirical analysis. Innovar: Revista de Ciencias Administrativas y Sociales, 23(50), 53-65.

Veugelers, W. (2011). Education and Humanism. Linking Autonomy and Humanity. Rotterdam: Sense Publishers.

Walter, S.G. \& Block, J. H. (2016). Outcomes of entrepreneurship education: An institutional perspective. Journal of Business Venturing, 31(2), 216-233. doi: /10.1016/j. jbusvent.2015.10.003

Woodside, A.G. (2010). Case study research: theory, methods, practice. Bingley, U.K: Emerald Group Pub. Ltd.

Yin, R.K. (2009). Case Study Research: Design and Methods. Thousand Oaks. CA: Sage.

Zhang, Y., Duysters, G. \& Cloodt, M. (2014). The role of entrepreneurship education as a predictor of university students' entrepreneurial intention. International Entrepreneurship and Management Journal, 10(3), 623-641. doi: 10.1007/s11365-012-0246-z

Fecha de recepción: 31 de octubre de 2016

Fecha de revisión: 31 de octubre de 2016

Fecha de aceptación: 23 de marzo de 2017 
